\title{
Prognostic Factors for Hepatocellular Carcinoma: 5 Years' Experience at the National Cancer Institute: A Retrospective Cohort Study
}

\author{
Hussein 0. Soliman ${ }^{1}$, Ahmed 0. Touny ${ }^{1}$, Reda H. Tabashy², \\ Mohamed M. Abou El Moaty 3 \\ ${ }^{1}$ Department of Surgical Oncology, National Cancer Institute, Cairo University, Giza City, Giza, Egypt \\ ${ }^{2}$ Department of Diagnostic and Interventional Radiology, National Cancer Institute, Cairo University, Giza City, Giza, Egypt \\ ${ }^{3}$ Department of Surgery, Mansoura University, Mansoura, Egypt \\ Email: ${ }^{\star}$ hus sein-soliman@nciegypt.org
}

How to cite this paper: Soliman, H.O., Touny, A.O., Tabashy, R.H. and El Moaty, M.M.A. (2018) Prognostic Factors for Hepatocellular Carcinoma: 5 Years' Experience at the National Cancer Institute: A Retrospective Cohort Study. Journal of Cancer Therapy, 9, 388-401.

https://doi.org/10.4236/jct.2018.95035

Received: February 27, 2018

Accepted: May 6, 2018

Published: May 9, 2018

Copyright $\odot 2018$ by authors and Scientific Research Publishing Inc. This work is licensed under the Creative Commons Attribution International License (CC BY 4.0).

http://creativecommons.org/licenses/by/4.0/

(C) (i) Open Access

\begin{abstract}
Background: Hepatocellular carcinoma is a great problem that poses a huge burden upon any healthcare system. In Egypt it has shown a doubling in its incidence rate. This study intended to assess the different prognostic factors for the overall survival of HCC patients in Egypt. Study Design: This is a retrospective patients' charts review of all patients (561) admitted to the National Cancer Institute (NCI), Cairo University with HCC from January 2007 to December 2011. The main outcome measures were the median overall survival. Also, all demographics, clinicopathological variables, laboratory data were tested as prognostic factors for the overall survival. Results: The median overall survival of our patients was seven months. The overall actuarial probability of survival during the follow-up period was $65 \%$ of patients at six months, $25 \%$ of patients at 12 months, $11 \%$ of patients at 18 months, and only $3 \%$ of patients at 24 months. In our study, a broad range of clinical, laboratory, tumor parameters, and demographic data of patients had shown statistical significance in univariate analysis. However, when all these significant factors in the univariate analysis were further analyzed by stepwise multivariate regression analysis which revealed that only age less than 60 , non-symptomatic cases, AFP less than 400, no PV thrombosis, no portal HTN, no ascites, no metastasis and potentially curative therapy were prognostic factors for the overall survival. Conclusion: Among all detected prognostic factors, only, management therapy is modifiable, other factors are non-modifiable. Thus, for HCC, the earlier the diagnosis and intervention, the better is the prognosis and outcome. Therefore, US screening and follow-up of high-risk groups are a must for early diagnose and better outcome.
\end{abstract}




\section{Keywords}

Prognostic Factors, Hepatocellular Cancer, HCV, AFP, Egypt

\section{Introduction}

Globally, hepatocellular carcinoma (HCC) remains one of the most prevalent malignancies. Liver cancer is principally a problem of the less developed regions where $83 \%$ of the new cancer cases worldwide occurred in 2012 (estimated 782,000 cases). It was the fifth most common cancer in men (7.5\% of the total) and the ninth in women (3.4\%). In 2012, it was the second most common cause of death from cancer worldwide, estimated to be responsible for about $(746,000$ deaths, $9.1 \%$ of the total). Hepatocellular Carcinoma (HCC) represents more than $90 \%$ of primary liver cancers and is a major global health problem [1]. In the past decade, its burden in Egypt has shown a doubling in the incidence rate; of course, this was because Egypt has got the highest prevalence of HCV all over the world [2].

Approximately $90 \%$ of cases of HCCs have a known underlying risk factor. The most frequent factors include chronic viral hepatitis (types B and C), alcohol intake, aflatoxin exposure, and of course cirrhosis [3]. Overall, one-third of cirrhotic patients will develop HCC during their lifetime. The risk is higher in patients with hepatitis infection. On the other hand, HCC arises in a cirrhotic background in up to $90 \%$ of cases [4].

In 2001, noninvasive diagnostic criteria for HCC based on a combination of imaging and laboratory findings had been reported for the first time by a panel of experts on HCC convened in Barcelona by European Association for the Study of the Liver (EASL) [5].

Management of HCC has many options. Surgery is the mainstay of treatment. Resection and transplantation can achieve the best outcomes for well-selected candidates [6]. Liver transplantation is the first treatment choice for patients with small multinodular tumors or those with single tumor $\leq 5 \mathrm{~cm}$ and advanced liver dysfunction. Theoretically, transplantation may simultaneously cure cancer and the underlying cirrhosis [7] cases at early stages of HCC who are not candidates for surgical therapies, local ablation either percutaneous or laparoscopic, are considered the first line treatment option [8].

Percutaneous ethanol injection (PEI) is a well-established technique for the treatment of nodular type HCC by achieving tumor necrosis while Radiofrequency ablation (RFA) is the most widely assessed alternative to PEI for local ablation of HCC. Although these treatments provide good results, they are unable to achieve response rates and outcomes comparable to surgery, even when applied as the first option [9].

Microwave ablation, laser ablation, and cryoablation have been and still under investigation. Trans-Arterial Chemoembolization (TACE) is the most widely 
used primary treatment for unresectable HCC and the recommended first-line therapy for patients at an intermediate stage of the disease [9].

A better understanding of the molecular carcinogenesis is critical for identifying novel targets and oncogenic addition loops in cases with HCC. Recently sorafenib, a multikinase inhibitor, has shown survival benefits in patients with advanced HCC. This advancement represents a breakthrough in the treatment of HCC and proves that molecular therapies can be useful in these cases.

Thus, the rationale intended for this study was to assess the different prognostic factors for the overall survival of HCC patients through the presentation of a 5-years' experience at the National Cancer Institute, Cairo University, Egypt.

\section{Patients and Methods}

This study was a retrospective patients' charts review of all patients admitted to the National Cancer Institute (NCI), Cairo University with HCC from January 2007 to December 2011.

This study followed the principles of the Declaration of Helsinki and in accordance with the Good Pharmacoepidemiology Practices (GPP) guidelines, and was approved by the medical ethical review committee of the institution. The data of the patients have presented anonymously with the protection of privacy and confidentiality.

\subsection{Case Definition}

The study population included the records of 561 HCC patients treated in the NCI during the study period. The diagnosis of HCC was based on the European Association for the Study of the Liver (EASL) and European Organization for Research and Treatment of Cancer (EORTC) 2012 [9]. A unique dynamic radiological behavior (contrast uptake in the arterial phase by CT, MRI, angiography or US) represented the backbone of radiological diagnosis of early HCC. In cirrhotic patients with nodules $>2 \mathrm{~cm}$, coincidental findings by two imaging techniques were considered diagnostic, or one imaging technique along with AFP levels above $400 \mathrm{ng} / \mathrm{ml}$. In all other circumstances, a biopsy was mandatory.

\subsection{Data Collection}

A standardized data sheet was administered to collect information on HCC parameters. Patients' records with the diagnosis of HCC during the period from January 2007 to December 2011 were retrieved from Biostatistics and Cancer Epidemiology Department at NCI. The following data were collected from the files:

Pre-Management data: Patients related factors like age, gender; clinical presentation and signs; co-morbidities (DM, Hypertension); liver function tests and Child score of the patient (child A, B, and C); and portal hypertension criteria (ascites and esophageal varices). Tumor-related factors collected were radiologic tumor criteria (site, size, the presence of cirrhosis ...); tumor markers (AFP); 
and tissue biopsy whenever available.

Post-Management data: Type of management either surgery (anatomical and non-anatomical resection and may be Right or Left lobe hepatectomy or extended hepatectomy); RadioFrequency (RF); Percutaneous Ethanol Injection (PEI); and Trans-Arterial Chemo-Embolization (TACE). Approach to management (Percutaneous or laparoscopic); histopathological tumor criteria (grade, satellites, microvascular invasion, safety margin, capsule); procedure associated morbidity (post-treatment liver failure, biliary leakage, wound infection or pulmonary complications); in-hospital mortality; and intensive care unit (ICU) and hospital stay.

\subsection{Main Outcome Measures}

The main outcome measures were the median overall survival (OS: the period from diagnosis of HCC until the death of the patient or last follow up). Also, all demographics, clinicopathological variables, laboratory data were tested as prognostic factors for the overall survival.

\subsection{Statistical Analysis}

Patient data were entered into computerized databases for clinical audit and quality improvement. The data were de-identified and entered into SPSS win statistical package version (V.20) for analysis. Patients with no retrievable information were excluded from the study.

This is a retrospective cohort study. Numerical data were expressed as the mean and standard deviation (SD) or median and range as appropriate. Categorical data were expressed as frequency and percentage. Survival analysis was done using Kaplan-Meier method. Comparison between two survival curves was made using the log-rank test; $\mathrm{p}$-value $\leq 0.05$ was considered significant. All tests were two-tailed.

\section{Results}

The study included 561 HCC patients treated in the NCI during the period from 2007 to 2011.

The median overall survival of our patients was seven months (Figure 1). The overall actuarial probability of survival during the follow-up period was $65 \%$ of patients at six months, $25 \%$ of patients at 12 months, $11 \%$ of patients at 18 months, and only $3 \%$ of patients at 24 months.

Finally, complete content and organizational editing before formatting. Please take note of the following items when proofreading spelling and grammar.

\subsection{Demographic Factors}

The majority of HCC patients (86.1\%) in this study were more than 50 years old with a mean age of 59 years. Around $70 \%$ of patients were male. All our cases developed HCC on top of cirrhosis, where $79.25 \%$ of patients were HCV positive, and $3.92 \%$ of them were HBV positive. 


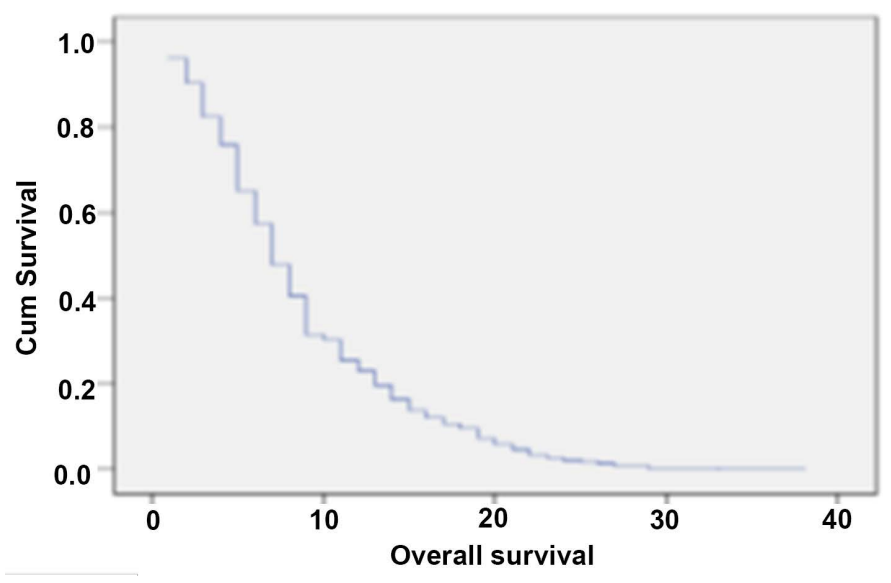

Figure 1. Kaplan-meier survival analysis of 561 patients.

Univariate analysis of overall survival according to demographic factors revealed that Age and gender have a highly significant impact. Median OS was higher in the age group from 50 - 60 than other groups. Also, median OS was higher in females than in males, as shown in Table 1.

\subsection{Clinical and Laboratory Data}

Most of HCC patients were symptomatic (75.4\%). The most prevalent presentation was abdominal pain and distention in $35.4 \%$ of patients, followed by jaundice in $28.3 \%$, ascites 6.7 , hematemesis $3 \%$, upper abdominal swelling $3.2 \%$, cachexia $1.7 \%$, liver cell failure $0.9 \%$ and dyspnea \& lower limb edema in $1 \%$ of patients.

The median OS in symptomatic cases (6 months) was significantly lower than in non-symptomatic cases (12 months), ( $\mathrm{p}<0.001)$.

More than $70 \%$ of patients were Child-Pugh score B or C. The median OS in cases with Child-Pugh A score $(11 \mathrm{~m})$ is significantly higher than in cases with Child-Pugh score B (7) or C (4), ( $\mathrm{p}<0.001)$, as shown in Figure 2.

According to the ECOG scale, $65.06 \%$ of patients had performance status PS2 or PS3 while $34.94 \%$ were PS1. The median OS in cases with PS1 (13 m) is significantly higher than in cases with PS2 (6) or PS3 (3), ( $p<0.001)$, as shown in Figure 3.

Patients with AFP more than 400 (66.67\%) had lower median OS (6 m) than those with AFP lower than or equal to 400, ( $\mathrm{p}<0.001$ ). The two-thirds of cases who have splenomegaly had a lower median OS $(6 \mathrm{~m})$ than the rest of cases $(9$ $\mathrm{m}),(\mathrm{p}<0.001)$. Esophageal varices were seen in $28.34 \%$ of patients; they had a lower median OS $(5 \mathrm{~m})$ than the rest of cases $(8 \mathrm{~m}),(\mathrm{p}<0.001)$. Those without ascites (35.29\%) had a higher median OS (12 $\mathrm{m})$ than other cases (7 months in mild \& 4 months in moderate to severe ascites), $(\mathrm{p}<0.001)$.

Those with bilirubin level less than $2(34.58 \%)$ had a higher median OS $(9 \mathrm{~m})$ than other cases ( 8 months in $2-3 \& 5$ months in cases with bilirubin $>3),(\mathrm{p}<$ 0.001). Also, those with albumin level more than 3.5 (30.66\%) had a higher 
Table 1. Univariate analysis of overall survival by patients' demographic data and risk factors.

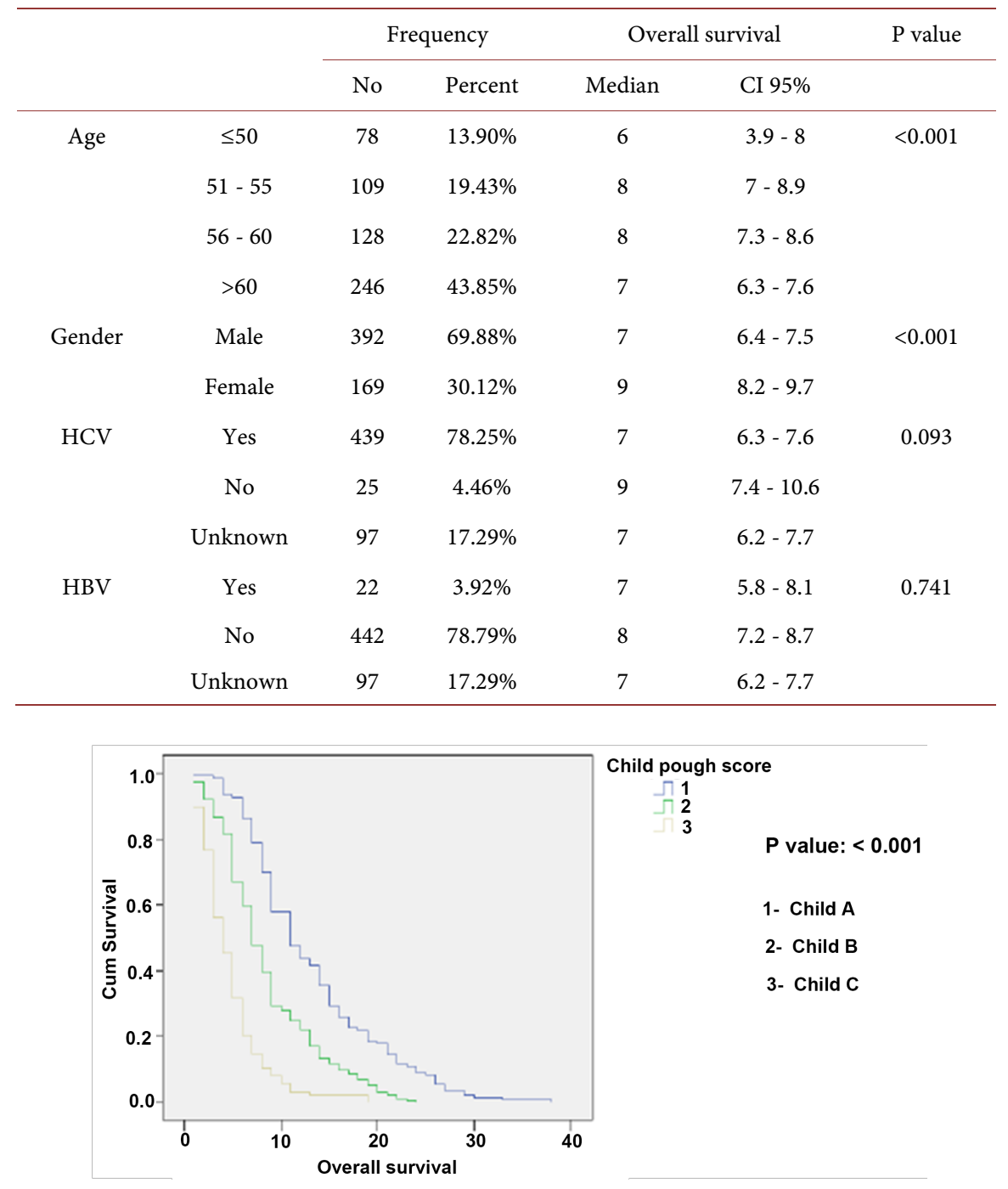

Figure 2. Kaplan-meier survival analysis according to child-pugh score.

median OS $(9 \mathrm{~m})$ than other cases, $(\mathrm{p}<0.001)$. Moreover, cases with normal alkaline phosphatase level $(47.06 \%)$ had a higher median OS (9 $\mathrm{m})$ than other cases $(6 \mathrm{~m}),(\mathrm{p}<0.001)$. Besides, cases with platelets count more than or equal to $100,000(47.06 \%)$ had a higher median OS $(9 \mathrm{~m})$ than other cases $(6 \mathrm{~m}),(\mathrm{p}<$ $0.001)$.

\subsection{Tumor Characteristics}

Patients with tumor size more than $5 \mathrm{~cm}$, either single $(7.31 \%)$ or multinodular (67.02\%), had a lower median OS $(6 \mathrm{~m})$ than other cases, $(\mathrm{p}<0.001)$.

The overall median survival for resectable tumors was 14 months and for non-resectable tumors was six months.

The radiological assessment had shown that; $70.8 \%$ of patients had positive tumor enhancement (contrast uptake in the arterial phase by CT, MRI with wash 


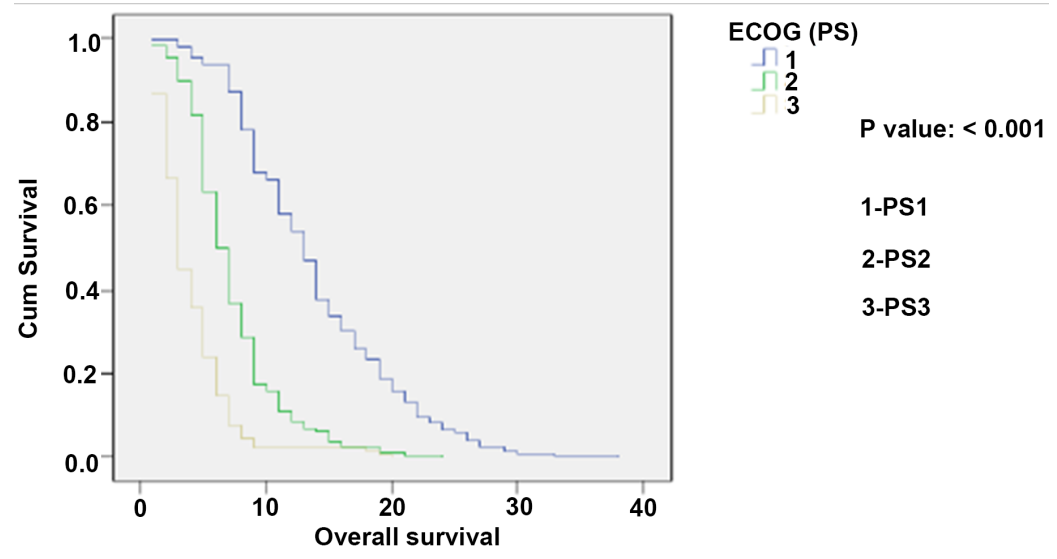

Figure 3. Kaplan-meier survival analysis according to EGOG status.

out in the venous phase), while $29.2 \%$ of them had no enhancement. However, positive tumor enhancement did not impact the median OS, $(\mathrm{p}=0.183)$.

Features of more advanced HCC like portal vein thrombosis, and distant metastases or porta hepatic lymph node involvement, were evident in $38.15 \%$, and $18.72 \%$ of patients respectively. Patients with portal vein thrombosis had a lower median OS $(5 \mathrm{~m})$ than other cases $(9 \mathrm{~m}),(\mathrm{p}<0.001)$. Also, patients with metastatic HCC had a lower median OS $(5 \mathrm{~m})$ than other cases $(8 \mathrm{~m}),(\mathrm{p}<0.001)$.

\subsection{Treatment Modalities}

Surgery was provided to $5.17 \%$ of patients with a perioperative mortality of $3.40 \%$ of resection cases. About $62.10 \%$ of resection patients had left hepatic lobectomy. Radiofrequency (RF) was provided to $9.45 \%$ of patients, with procedure-associated mortality of $1.9 \%$ of cases. Also, $32.1 \%$ of RF cases were subjected to further management either TACE or chemotherapy.

TACE was provided to $31.19 \%$ of cases, where all patients had from 1 - 3 cycles of conventional TACE, with embolization of the feeding hepatic artery after local injection of combination chemotherapy of cisplatin and adriamycin. Procedure-associated mortality was $2.9 \%$ of cases. Systemic chemotherapy (Sorafenib) was provided to $14.26 \%$ of cases, also was given to a minority of cases had either RF or TACE.

Supportive symptomatic care represented the primary line of care in 39.93\% of patients, and the inevitable end stage following all other procedures.

Univariate analysis of overall survival according to therapy (Table 4), showed that the median OS for patients subjected to surgery was 22 months, radiofrequency 18 months, TACE 10 months, Chemotherapy 7 months, and for patients with supportive care it was four months (Figure 4).

\subsection{Multivariate Analysis}

All significant factors in the aforementioned univariate analysis were further analyzed by stepwise multivariate regression analysis (Table 5). The multivariate analysis revealed that only the following are prognostic factors: 


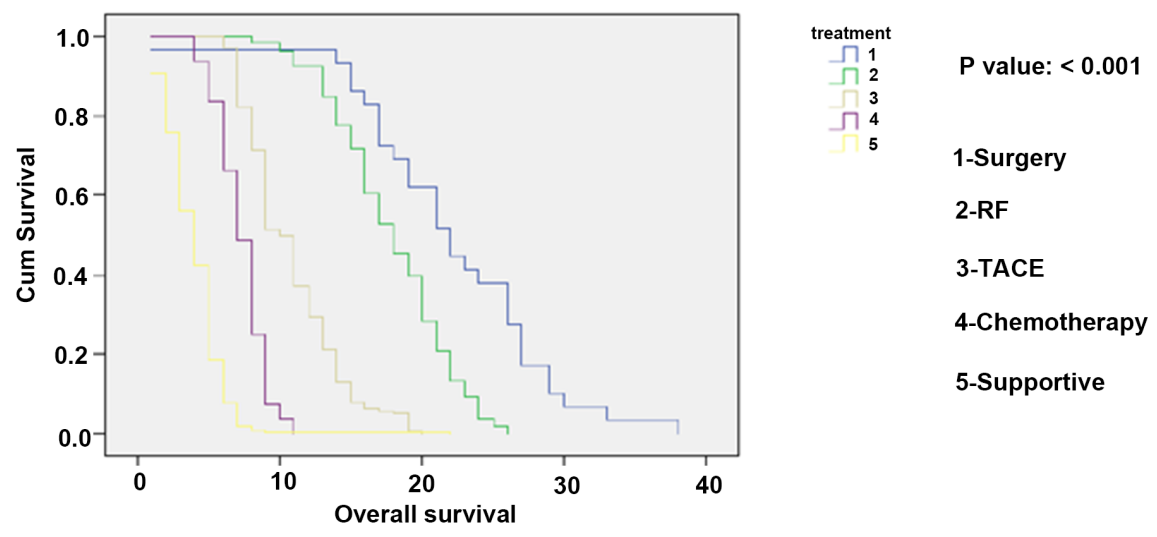

Figure 4. Kaplan-Meier survival analysis according to type of treatment.

Age less than 60, non-symptomatic cases, AFP less than 400, no PV thrombosis, no portal HTN, no ascites, no metastasis and potentially curative therapy, as shown in Table 5.

\section{Discussion}

In the current retrospective cohort study, we studied the pattern of HCC in Egypt as regards the factors affecting the overall survival.

According to the data of the National Cancer Registry Program of Egypt from 2008-2011, liver cancer is the most predominant cancer in Egypt.

HCC secondary to cirrhosis is a complex and heterogeneous disease with wide variations during its clinical course. Different staging system had been proposed to predict prognosis, but better knowledge of the natural history of the tumor as well as the development of clinically based staging systems as the BCLC classification, has meant that life expectancies can be confidently predicted, and the appropriate treatment can be chosen according to the stage.

Today, the diagnosis of HCC is established within the national surveillance programs in developed countries, while the diagnosis of symptomatic advanced stage disease, remains the characteristic of underdeveloped countries, and finally grave prognosis.

Moreover, HCC is a significant burden for healthcare systems in the developing countries with $84 \%$ of the world HCC cases, with the highest annual fatality ratio of any human malignancy (0.96). Also, underdeveloped regions may even have a 100-fold higher incidence of HCC compared to developed countries, when considering that many HCC cases remain under-diagnosed or under-reported.

The current study is a population-based study of the extent and determinants of HCC in Egypt, and the outcomes of different ways of management. Several Western and Eastern trials had been reported on similar cohorts of patients to assess the natural course of the disease, prognostic factors, and best management modalities which prolong survival.

Our study included 561 HCC patients with a mean age of 59.6 years old. Based 
on the study of Tanaka et al. (2008), the mean age of patients with HCC is appreciably younger in Chinese and in black African populations ranging from 50 - 55 years, in contrast to Japan, where the incidence of HCC is highest in the cohort of men aged 70 - 79 years.

We had strong male preponderance, with a male to female ratio of (2.3), which was nearly similar to that reported by (IARC 2011), where male to female ratio estimated to be (2.4).

All our cases developed HCC on top of cirrhosis, with HCV infection as the principal risk factor. Worldwide there is no doubt of cirrhosis being the most substantial single risk factor present in $80 \%-90 \%$ of HCC cases, and [4]. The vast difference is that cirrhosis is the underlining risk factor. In China, where $\mathrm{HBV}$ is predominant, studies reported HBV infection in more than $70 \% \mathrm{HCC}$ cases. In contrast to the USA, where alcoholic abuse $(22.5 \%$ of patients) comes first, followed by HCV infection (16.5\%) and HBV infection (8.8\%). Meanwhile, in Europe Winkel et al. (2012) reported chronic alcohol abuse as the most common single risk factor in $44 \%$ of patients followed by HCV infection in $18.8 \%$ of patients and a minority of HBV infection. All these studies agreed with the apparent geographical distribution nature of HCC claimed by Parkin et al. (2005) [3].

About $75.4 \%$ of our cases were symptomatic, and $24.6 \%$ of cases accidentally discovered during an investigation for another complaint. Based on studies done by Okuda et al. (1985), and Cabibbo et al. (2010) the presence of symptoms, is one of the main poor clinical prognostic factors in HCC patients.

There were certain features characterized our cohort and could explain our results. For instance, during clinical assessment of our patient we found that nearly $74 \%$ of our patients were classified as child B \& C cirrhosis, and about $65 \%$ of them presented with an ECOG of (2 - 5) scale. In contrast, El Serag et al. (2006) study on 2963 American HCC patients during the period from 1992 to 1999 reported that only $13 \%$ of patients were classified as child C cirrhosis. Also, Winkel et al. (2012) in their study on 405 German HCC patients during the period from 1998 to 2009 showed that more than half of the patients were classified as child A cirrhosis; most of the patients were in a good or fairly good general condition at time of HCC diagnosis. Even the study of Cabibbo et al. (2012) on 320 untreatable Italian HCC patients showed that $33 \%$ of them were child A cirrhosis.

Another finding with the radiological assessment of our patients, only $3 \%$ of them presented with lesions $\leq 2 \mathrm{~cm}$, Resection ability was applicable in $26 \%$ of patients. That resembles, to great extent, the features reported by El Serag et al. (2006), and Winkel et al. (2012) studies; where $30 \%$ and $24 \%$ of lesions were within the resection limit, respectively. However, these studies represented an earlier era.

On the other hand, EASL guidelines (2012) and Llovet et al. (2008) reported that tumors less than $2 \mathrm{~cm}$ in diameter currently represent up to $30 \%$ of cases in Japan. Also, they reported that early HCC diagnosis is feasible in $30 \%-60 \%$ of 
cases in the developed countries, which enables the application of curative treatments [9].

Advanced disease stage of our cohort was remarkable as $38.1 \%$ of cases presented with portal vein thrombosis. Also, metastasis and porta hepatic lymph node enlargement were evident in $18.5 \%$ of cases. Metastatic disease was also reported in $16 \%$ of El Serag et al. (2006) cohort, in contrary to Winkel et al. (2012) cohort, where portal vein thrombosis and metastasis were evident in only $20.1 \%$ and $6.4 \%$ of cases, respectively. Even in Cabibbo et al. (2012) cohort, portal vein thrombosis did not reach our score and was evident in $31 \%$ of cases.

Following the advanced nature of our patients, it was an ordinary finding that AFP level $\geq 400 \mathrm{ng} / \mathrm{ml}$ was evident in $66.6 \%$ of patients. In contrast, Winkel et al. (2012) showed that $25 \%$ of cases had AFP level $\geq 423 \mathrm{ng} / \mathrm{ml}$. Also, Cabibbo et al. (2012) reported only $24 \%$ of patients presented with AFP level $\geq 200 \mathrm{ng} / \mathrm{ml}$. Surprisingly, in our study, we had the following AFP levels (216,000, 143,000, 96,000 , and $87,000 \mathrm{ng} / \mathrm{ml}$ ).

And when we came to treatment modalities; despite that $26 \%$ of our cases were within the resection limit, potentially curative treatment (surgery and RF) was applied to only $14 \%$ of them, and supportive care had represented the main line of care in $40 \%$ of cases. That coincide apparently to that reported by El Serag et al. (2006) study, where $13 \%$ of patients had curative treatment, but when taking into consideration that $68 \%$ of them had a resection and $7 \%$ of them had transplantation, there was a difference. Also, when we came back to Winkel et al. (2012) study, we found that $24 \%$ of patients subjected to curative treatment, which is the same percentage of patients within resection limit in other studies. There are many possible explanations for this obvious underutilization of HCC therapy. These are the severity of liver disease, comorbid illness, and functional status which are important determinants of treatment and prognosis of HCC patients.

Median overall survival of our patients was seven months which is considered low. The results of El Serag et al. (2006) study had shown a low overall median survival of 3.5 months for his cohort. In fact, both studies results are comparable with studies on untreatable HCC like Yeung et al. (2005) study, which reported a three months median overall survival, and also Cabibbo et al. (2012) study, which reported 6.8 months for his untreatable cohort. However, when we came to potentially curable cohorts like that of Winkel et al. (2012) study, and Xu et al. (2015) study, we found that median overall survival had increased to 18.1 months and 25.2 months, respectively.

Identification of prognostic factors within a given study population is the basis on which all studies had been made, and staging systems had been developed. In our study, a broad range of clinical, laboratory, tumor parameters, and demographic data of patients had shown statistical significance in univariate analysis (Tables 1-4). However, in multivariate analysis; age, patients symptomatology, AFP level, tumor characters, portal hypertension, the presence of ascites, 
Table 2. Univariate analysis of overall survival by patients' clinical and laboratory data.

\begin{tabular}{|c|c|c|c|c|c|c|}
\hline & & \multicolumn{2}{|c|}{ Frequency } & \multicolumn{2}{|c|}{ Overall survival } & \multirow[b]{2}{*}{$P$ value } \\
\hline & & No & Percent & Median & CI 95\% & \\
\hline \multirow[t]{2}{*}{ Symptomatic } & No & 138 & $24.60 \%$ & 12 & $10.4-13.5$ & $<0.001$ \\
\hline & Yes & 423 & $75.40 \%$ & 6 & $5.5-6.4$ & \\
\hline \multirow[t]{3}{*}{ Child Pugh score } & A & 148 & $26.38 \%$ & 11 & $9.3-12.6$ & $<0.001$ \\
\hline & B & 275 & $49.02 \%$ & 7 & $6.4-7.5$ & \\
\hline & $\mathrm{C}$ & 138 & $24.60 \%$ & 4 & $3.3-4.6$ & \\
\hline \multirow[t]{3}{*}{ ECOG scale } & PS1 & 196 & $34.94 \%$ & 13 & $11.9-14$ & $<0.001$ \\
\hline & PS2 & 236 & $42.07 \%$ & 6 & $5.5-6.4$ & \\
\hline & PS3 & 129 & $22.99 \%$ & 3 & $2.6-3.3$ & \\
\hline \multirow[t]{3}{*}{ AFP } & $<20$ & 57 & $10.16 \%$ & 9 & $8.4-9.5$ & $<0.001$ \\
\hline & $20-400$ & 130 & $23.17 \%$ & 11 & $9.5-12.4$ & \\
\hline & $>400$ & 374 & $66.67 \%$ & 6 & $5.4-6.5$ & \\
\hline \multirow[t]{2}{*}{ Splenomegaly } & Yes & 374 & $66.67 \%$ & 6 & $5.4-6.5$ & $<0.001$ \\
\hline & No & 187 & $33.33 \%$ & 9 & $8.4-9.5$ & \\
\hline \multirow[t]{2}{*}{ Portal HTN } & Yes & 295 & $52.58 \%$ & 6 & $5.5-6.4$ & $<0.001$ \\
\hline & No & 266 & $47.42 \%$ & 9 & $8-9.9$ & \\
\hline \multirow[t]{2}{*}{ Esophageal varices } & Yes & 159 & $28.34 \%$ & 5 & $4.3-5.6$ & $<0.001$ \\
\hline & No & 402 & $71.66 \%$ & 8 & $7.4-8.5$ & \\
\hline \multirow[t]{3}{*}{ Ascites } & Non & 198 & $35.29 \%$ & 12 & $10.7-13.2$ & $<0.001$ \\
\hline & Mild & 232 & $41.35 \%$ & 7 & $6.4-7.5$ & \\
\hline & Moderate to Severe & 131 & $23.35 \%$ & 4 & $3.2-4.7$ & \\
\hline \multirow[t]{3}{*}{ Bilirubin } & $<2$ & 194 & $34.58 \%$ & 9 & $8-9.9$ & $<0.001$ \\
\hline & From 2 - 3 & 236 & $42.07 \%$ & 8 & $7.3-8.6$ & \\
\hline & $>3$ & 131 & $23.35 \%$ & 5 & $4.3-5.7$ & \\
\hline \multirow[t]{3}{*}{ Albumin } & $\geq 3.5$ & 172 & $30.66 \%$ & 9 & $8.2-9.7$ & $<0.001$ \\
\hline & $2.8-3.5$ & 285 & $50.80 \%$ & 7 & $6.2-7.7$ & \\
\hline & $<2.8$ & 104 & $18.54 \%$ & 5 & $6.5-7.4$ & \\
\hline \multirow[t]{2}{*}{ Platelets } & $\geq 100,000$ & 325 & $57.93 \%$ & 9 & $7.9-10$ & $<0.001$ \\
\hline & $<100,000$ & 236 & $42.07 \%$ & 5 & $4.5-5.5$ & \\
\hline \multirow[t]{2}{*}{ AL.PH } & Normal & 264 & $47.06 \%$ & 9 & $8-9.9$ & $<0.001$ \\
\hline & Elevated & 297 & $52.94 \%$ & 6 & $5.5-6.4$ & \\
\hline
\end{tabular}

evidence of portal vein thrombosis, the presence of metastasis, and type of management could be defined as independent prognostic factors with statistical significance (Table 5).

Based on data reporting the natural history of the disease by Okuda et al. (1985) and Cabibbo et al. (2010), the main clinical prognostic factors for HCC 
Table 3. Univariate analysis of overall survival by tumor characteristics.

\begin{tabular}{|c|c|c|c|c|c|c|}
\hline & & \multicolumn{2}{|c|}{ Frequency } & \multicolumn{2}{|c|}{ Overall survival } & \multirow[t]{2}{*}{$P$ value } \\
\hline & & No & Percent & Median & CI 95\% & \\
\hline \multirow{5}{*}{$\begin{array}{c}\text { Tumor } \\
\text { characteristics }\end{array}$} & Single $\leq 2 \mathrm{~cm}$ & 17 & $3.03 \%$ & 8 & $0.00-18.7$ & \\
\hline & Single $2-5 \mathrm{~cm}$ & 114 & $20.32 \%$ & \multirow[b]{2}{*}{15} & \multirow[b]{2}{*}{$13.2-16.7$} & $<0.001$ \\
\hline & 3 nodules $\leq 3 \mathrm{~cm}$ & 13 & $2.32 \%$ & & & \\
\hline & Single $>5 \mathrm{~cm}$ & 41 & $7.31 \%$ & \multirow[b]{2}{*}{6} & \multirow[b]{2}{*}{$5.5-6.5$} & \\
\hline & Multinodular $>5 \mathrm{~cm}$ & 376 & $67.02 \%$ & & & \\
\hline \multirow{2}{*}{$\begin{array}{c}\text { Tumor } \\
\text { enhancement }\end{array}$} & Yes & 395 & $70.41 \%$ & 7 & $6.4-7.5$ & \multirow[t]{2}{*}{0.183} \\
\hline & No & 166 & $29.59 \%$ & 7 & $6.1-7.8$ & \\
\hline \multirow{2}{*}{ PV thrombosis } & Yes & 214 & $38.15 \%$ & 5 & $4.6-5.3$ & \multirow[t]{2}{*}{$<0.001$} \\
\hline & No & 347 & $61.85 \%$ & 9 & $8.1-9.8$ & \\
\hline \multirow{2}{*}{ Metastasis } & Yes & 105 & $18.72 \%$ & 5 & $4.5-5.4$ & \multirow[t]{2}{*}{$<0.001$} \\
\hline & No & 456 & $81.28 \%$ & 8 & $7.4-8.5$ & \\
\hline
\end{tabular}

Table 4. Univariate analysis of overall survival by treatment modalities.

\begin{tabular}{cccccccc}
\hline & \multicolumn{2}{c}{ Frequency } & \multicolumn{2}{c}{ Overall Survival } & One-month mortality & p value \\
\cline { 2 - 8 } & No & Percent & Median & CI 95\% & No & Percent & \\
\hline Surgery & 29 & $5.17 \%$ & 22 & $18.8-25.1$ & 1 & $3.40 \%$ & $<0.001$ \\
Radiofrequency & 53 & $9.45 \%$ & 18 & $16-19.9$ & 1 & $1.90 \%$ & \\
TACE & 175 & $31.19 \%$ & 10 & $9.3-10.6$ & 5 & $2.90 \%$ & \\
Chemotherapy & 80 & $14.26 \%$ & 7 & $6.4-7.5$ & 6 & $7.50 \%$ & \\
Supportive & 224 & $39.93 \%$ & 4 & $3.5-4.4$ & 21 & $9.40 \%$ & \\
\hline
\end{tabular}

Table 5. Multivariate analysis of prognostic factors of HCC survival in study patients.

\begin{tabular}{cccccc}
\hline & \multicolumn{2}{c}{$\begin{array}{c}\text { Unstandardized } \\
\text { Coefficients }\end{array}$} & $\begin{array}{c}\text { Standardized } \\
\text { Coefficients }\end{array}$ & $\mathrm{t}$ & p value \\
\cline { 2 - 5 } & $\mathrm{B}$ & Std. Error & $\mathrm{B}$ & & \\
\hline Age less than 60 & 0.03 & 0.02 & 0.19 & 2.01 & 0.045 \\
Non-symptomatic & 1.61 & 0.40 & 0.20 & 4.03 & 0.000 \\
AFP less than 400 & 0.96 & 0.25 & 0.24 & 3.81 & 0.000 \\
No PV thrombosis & 2.38 & 0.40 & 0.38 & 5.96 & 0.000 \\
No portal HTN & 3.48 & 0.36 & 0.50 & 9.73 & 0.000 \\
No ascites & 0.84 & 0.39 & 0.16 & 2.14 & 0.033 \\
No metastasis & 2.25 & 0.43 & 0.39 & 5.17 & 0.000 \\
Non-curative therapy & -2.80 & 0.22 & -1.03 & -12.64 & 0.000 \\
\hline
\end{tabular}


patients are related to the tumor status (number and size of nodules, the presence of vascular invasion \& extrahepatic spread). That was followed by liver function (Child-Pugh's class, bilirubin, albumin, portal hypertension \& ascites) and general health status (ECOG classification and the presence of symptoms). Etiology has not been identified as an independent prognostic factor. And that was nearly compatible with our study, except for age and AFP level. Xu et al. (2015) had reported a comparison study between young and aged Patients with HCC. They found that the younger HCC patients shared the similar liver conditions with older cases. Regarding tumor characteristics, the AFP level in the younger group was significantly higher, indicating the more aggressiveness of the tumors in young patients. However, irrespective of the aggressiveness of the tumor state, younger patients still tend to have a better prognosis, probably due to the better health conditions, and stronger tolerance to curative treatment.

As regard AFP, Llovet et al. (2008) reported that AFP levels, VEGF and Ang had been shown to be independent prognostic value in large cohorts of untreated advanced tumors. It had been shown to predict the risk of drop-out in patients on the waiting list for liver transplantation, response to local ablation, response to loco-regional therapies, and in the outcome of advanced tumors.

Finally, we could suggest that absence of screening high-risk groups and late diagnosis are the main determinant of our grave outcome. Reluctance in diagnose, advanced nature disease, associated comorbidities, bad performance status, apparent underutilization of HCC therapy, and finally grave prognosis characterize HCC in Egypt.

\section{Conclusion}

Among all detected prognostic factors, only, management therapy is modifiable, other factors are non-modifiable. Thus, for HCC, the earlier the diagnosis and intervention, the better is the prognosis and outcome. Therefore, US screening and follow-up of high-risk groups are a must for early diagnose and better outcome.

\section{References}

[1] Malik, A.S., Boyko, O., Atkar, N. and Young, W.F. (2001) A Comparative Study of MR Imaging Profile of Titanium Pedicle Screws. Acta Radiologica, 42, 291-293. https://doi.org/10.1080/028418501127346846

[2] Hu, T. and Desai, J.P. (2004) Soft-Tissue Material Properties under Large Deformation: Strain Rate Effect. Proceedings of the 26th Annual International Conference of the IEEE EMBS, 1-5 September 2004, San Francisco, 2758-2761.

[3] Ortega, R., Loria, A. and Kelly, R. (1995) A Semiglobally Stable Output Feedback PI2D Regulator for Robot Manipulators. IEEE Transactions on Automatic Control, 40, 1432-1436. https://doi.org/10.1109/9.402235

[4] Wit, E. and McClure, J. (2004) Statistics for Microarrays: Design, Analysis, and Inference. 5th Edition, John Wiley \& Sons Ltd., Chichester.

https://doi.org/10.1002/0470011084 
[5] Prasad, A.S. (1982) Clinical and Biochemical Spectrum of Zinc Deficiency in Human Subjects. In: Prasad, A.S., Ed., Clinical, Biochemical and Nutritional Aspects of Trace Elements, Alan R. Liss, Inc., New York, 5-15.

[6] Giambastiani, B.M.S. (2007) Evoluzione Idrologicaed Idrogeologica Della Pineta di san Vitale (Ravenna). Ph.D. Thesis, Bologna University, Bologna.

[7] Wu, J.K. (1994) Two Problems of Computer Mechanics Program System. Proceedings of Finite Element Analysis and CAD, Peking University Press, Beijing, 9-15.

[8] Honeycutt, L. (1998) Communication and Design Course. http://dcr.rpi.edu/commdesign/class1.html

[9] Wright and Wright, W. (1906) Flying-Machine. US Patent No. 821393. 